\title{
Advances in Hemodynamic Monitoring in Heart Failure Patients
}

\author{
Teruhiko Imamura $^{1}$ and Nikhil Narang ${ }^{2}$
}

\begin{abstract}
:
Abnormal hemodynamics are associated with poor clinical outcomes in patients with heart failure. Given the invasiveness and unfeasibility of routine right heart catheterization, non-invasive methodologies to monitor hemodynamics are needed. The CardioMEMS ${ }^{\mathrm{TM}}$ device is a recently developed technology that enables remote monitoring of pulmonary artery pressures via an implanted sensor located in the distal left pulmonary artery. Along with the CardioMEMS ${ }^{\mathrm{TM}}$, a remote dielectric system might be another promising device that reports indirect intra-thoracic filling pressures associated with pulmonary congestion. In patients with advanced heart failure who require left ventricular assist devices, non-invasive hemodynamic monitoring is particularly necessary, given the increased procedural risks of invasive assessments. The HeartWare ventricular assist device flow waveform is a variable under active investigation that might also provide an estimation of filling pressures. This methodology is expected to lead to the development of a smart pump that can adjust its own settings, including the pump speed, automatically by monitoring the hemodynamics.
\end{abstract}

Key words: right heart catheterization, ventricular assist device, congestion

(Intern Med 60: 167-171, 2021)

(DOI: 10.2169/internalmedicine.4615-20)

\section{Introduction}

Over the last two decades, the implication of hemodynamic measurements in the management of heart failure (HF) patients has been a topic of great debate (1). The role of hemodynamic-guided therapies was investigated in the Evaluation Study of Congestive HF and Pulmonary Artery Catheterization Effectiveness (ESCAPE) trial (2), in which right heart catheterization (RHC)-guided therapies did not improve the short-term outcomes of patients with acute decompensated heart failure (ADHF). The study led to a significant reduction in the use of routine RHC in patients presenting with ADHF, despite the study's limited inclusion criteria, which do not routinely apply to contemporary patient populations with advanced HF.

However, a post-hoc analysis of the ESCAPE trial found that a persistently elevated cardiac filling pressures posttreatment was strongly associated with worse clinical outcomes (3). These findings underscore the importance to identify high-risk hemodynamic profiles. Furthermore, the clinical assessment of patients with chronic, advanced HF without an invasive hemodynamic assessment may lead to the delayed identification of high-risk hemodynamic phenotypes.

Although the precise data from invasive hemodynamics are the preferred way of evaluating HF patients, the primary risk lies in the invasiveness of the procedure and associated risk of complications, despite their rarity. In addition, continuous monitoring of hemodynamics in the inpatient setting is challenging and costly, often requiring a stay in the intensive-care unit. In contrast, static hemodynamic measurements have short-term usefulness, although a patient's clinical course can change dramatically, ultiamtely requiring subsequent continuous hemodynamic monitoring.

Given these limitations of traditional RHC and the high burden of disease-associated morbidity with HF, an approach to non-invasive remote hemodynamic monitoring is needed. We herein review several recently developed technologies that monitor hemodynamic parameters in both HF patients and those with left ventricular assist devices (LVADs).

${ }^{1}$ Second Department of Medicine, University of Toyama, Japan and ${ }^{2}$ Advocate Christ Medical Center, USA 
A. B.

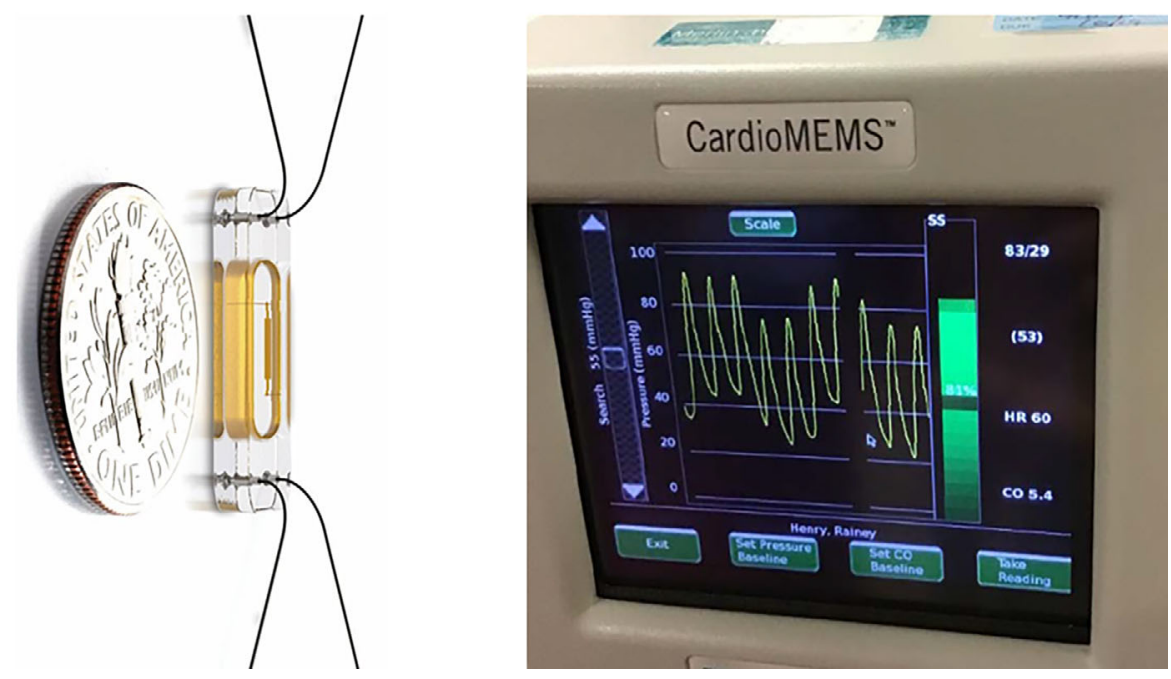

Figure 1. CardioMEMS ${ }^{\mathrm{TM}}$ device (A). The actual monitor of the pulmonary artery pressure obtained from a CardioMEMS ${ }^{\mathrm{TM}}$ located in the pulmonary artery $(\mathrm{B})$. Reused with permission of $\mathrm{Ab}$ bott.

\section{Pulmonary artery pressure monitoring (CardioMEMS ${ }^{\mathrm{TM}}$ )}

The CardioMEMSTM is a recently developed remote hemodynamic monitoring device (4), now used often in daily clinical care in both Europe and the United States. It comprises a small sensor designed to be implanted in the major pulmonary artery via RHC, which subsequently allows for remote pulmonary artery pressure (PAP) monitoring (Fig. 1A). In 2014, the CardioMEMS ${ }^{\mathrm{TM}}$ received approval from the Food and Drug Administration for the monitoring of PAP in patients with New York Heart Association functional class III symptomology who had been hospitalized for HF (5). CardioMEMSTM implantation also carries a class IIb recommendation from the European Society of Cardiology to monitor PAP in symptomatic patients with HF with a history of HF hospitalization (6).

The CardioMEMS ${ }^{\mathrm{TM}}$ device is most commonly placed in the left distal pulmonary artery percutaneously via an overthe-wire system following an RHC in the cardiac catheterization laboratory. After pressure calibration, a continuous PAP waveform can be visualized remotely on the monitor (Fig. 1B). In general, the PAP is measured by the patient once a day in the supine position, over the external antenna that activates the implanted CardioMEMS ${ }^{\mathrm{TM}}$ sensor. Wirelessly delivered PAP measurements are typically checked once or more per week by clinicians, triggering data-driven adjustments in HF-specific therapies, including titration of medications. The diastolic PAP is frequently used as a surrogate for the pulmonary capillary wedge pressure (PCWP) except in scenarios of pulmonary arterial hypertension, in which diastolic PAP is sometimes inappropriately high over PCWP (7).

Recently, in the CardioMEMS ${ }^{\mathrm{TM}}$ Heart Sensor Allows
Monitoring of Pressure to Improve Outcomes in NYHA Class III HF Patients (CHAMPION) trial (8), the use of a CardioMEMS $^{\mathrm{TM}}$ led to a $30 \%$ reduction in HF readmissions, equating to massive reductions in healthcare expenditures and reduced disease-specific morbidity. This outcome was likely due to a higher rate of adjustments of diuretic therapy and neurohormonal blockade agents in the group with active hemodynamic monitoring (9).

Of note, this device has not yet been approved in Japan. One of the limitations of this technology would be how to monitor the data and how to let patients change medications.

\section{Pulmonary congestion monitoring [Remote Dielec- tric System (ReDS)]}

ReDS employs low-power electromagnetic signals emitted between two sensors (one each on the anterior and posterior body surfaces) embedded in a wearable vest (Fig. 2A) (10). The analyzed signal reflects the dielectric properties of the portion of the lung between the sensors. The dielectric coefficient of a material is represented by a frequency-dependent complex number describing its interaction with electromagnetic energy, including the degrees of absorption, reflection, and transmission of the energy. Given water has a very high dielectric coefficient and air has a very low dielectric constant, the dielectric coefficient of tissue is determined predominantly by its fluid content. This measurement takes 90 seconds to obtain.

We recently showed that the ReDS measurement correlated with the actual PCWP measured by RHC (Fig. 2B) (11). Given the high sensitivity and moderate specificity, we suggest using ReDS technology to screen for pulmonary congestion in the ambulatory setting. Invasive assessments, including RHC, should be considered when pa- 


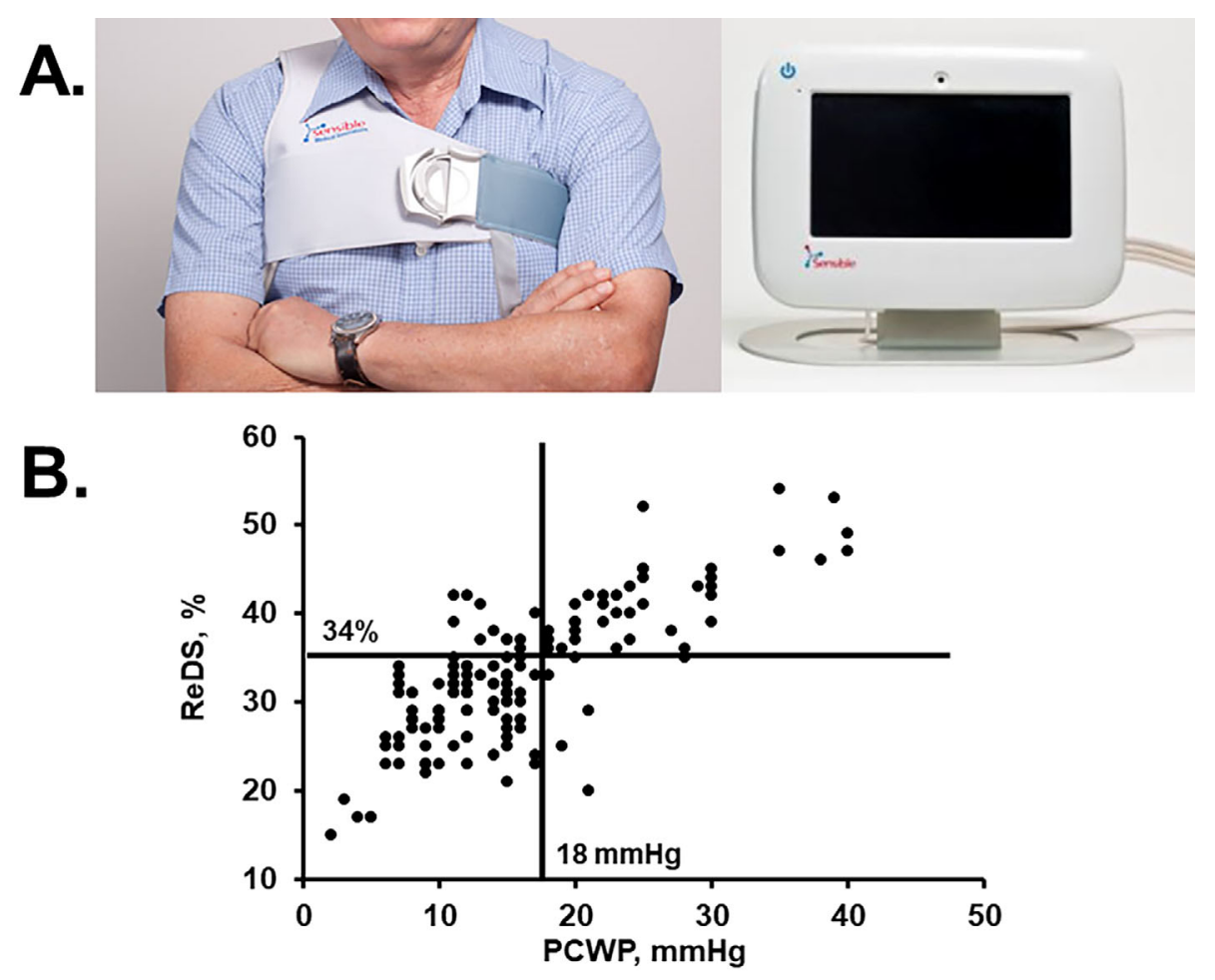

Figure 2. Remote dielectric System (A). Correlation between the actual measured pulmonary capillary wedge pressure and ReDS with each cut-off (B). Reused from reference 11 with permission. ReDS: Remote Dielectric System, PCWP: pulmonary capillary wedge pressure

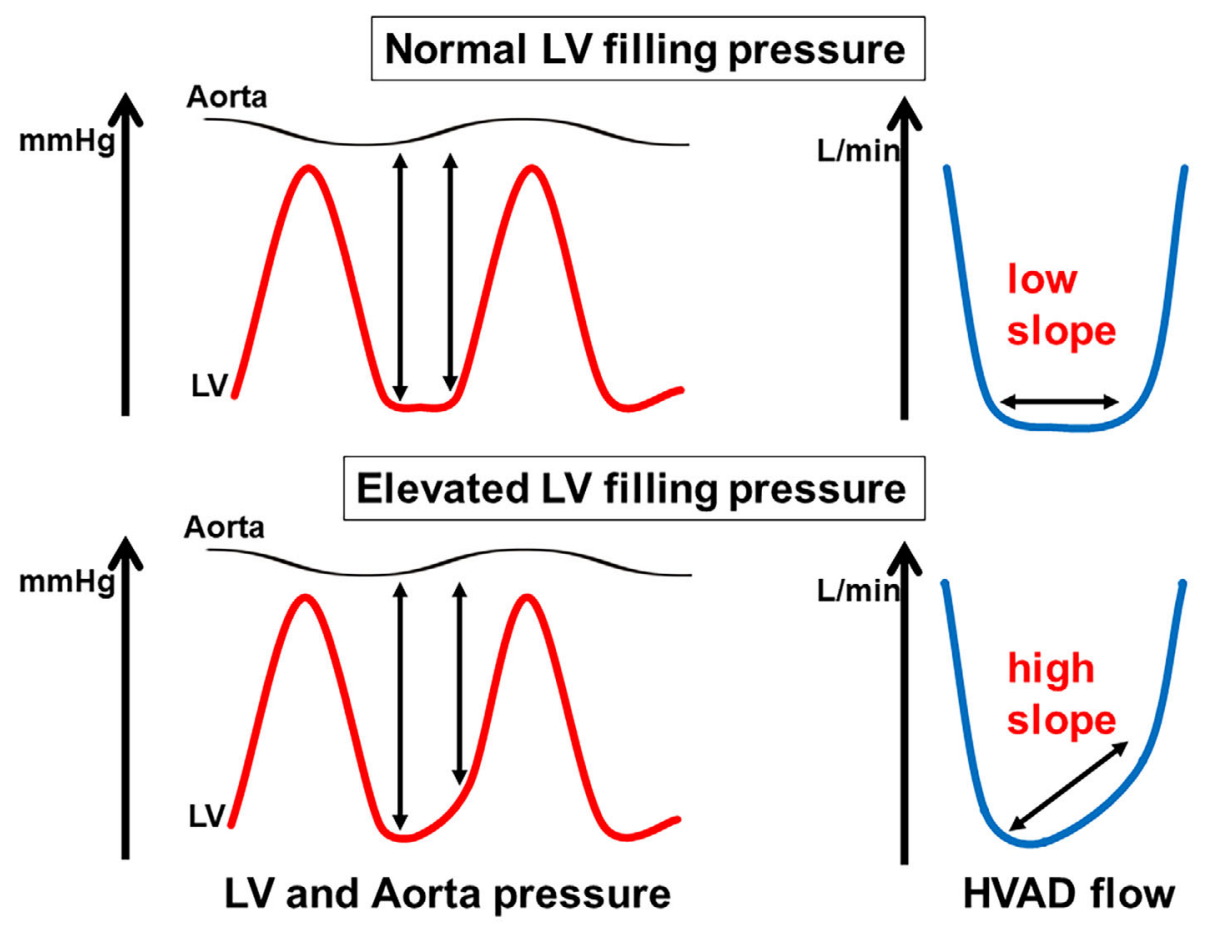

Figure 3. Relationship between the actual left ventricular filling pressure (red line) and the diastolic slope of HeartWare LVAD flow waveform. HeartWare LVAD pump flow depends on the pressure gradient between the left ventricle and aorta. When the pressure gradient drops, the pump flow increases. Therefore, when the left ventricular filling pressure increases in decompensated $\mathrm{HF}$, the pressure gradient decreases, and the diastolic slope of the pump flow becomes increases. LVAD: left ventricular assist device, $\mathrm{HF}$ : heart failure 
tients have ReDS above $34 \%$ (cut-off to estimate PCWP $>18 \mathrm{mmHg}$ ) to identify high-risk patients who require more intensive treatments. As shown in the moderate specificity of this technology, an elevated ReDS value does not always indicate pulmonary congestion. The ReDS value might also be increased in other clinical situations that increase the fluid level in the lung, including pulmonary pneumonia. Further prospective trials are needed to clarify whether or not ReDS-guided management improves the clinical outcomes in HF management (12).

\section{Hemodynamic monitoring in LVAD patients}

Hemodynamics measurements are also critically important in patients with durable mechanical assist devices (1). Recently, it was shown that hemodynamic measurements are not necessarily optimized following LVAD implantation in stable ambulatory patients (13). Furthermore, abnormal hemodynamics in LVAD patients are associated with worse clinical outcomes, including $\mathrm{HF}$ readmission and hemocompatibility-related adverse events (gastrointestinal bleeding, stroke, and pump thrombosis) (14).

Invasive hemodynamic optimization studies for LVAD patients combining simultaneous RHC and echocardiography to assess stepwise changes in filling pressures and ventricular size/valve opening (RAMP study) may allow the clinician to choose a device speed that best suits the patient. We recently demonstrated in a prospective randomized control trial that patients who underwent hemodynamic ramp-guided management had reduced readmissions and a better quality of life than the control group with conventional management due to hemodynamic improvement driven by pump speed adjustment and medication titration (Ramp-it-Up trial) (15).

However, performing serial RHCs should be cautioned in LVAD patients, given the increased risk of bleeding due to a need for uninterrupted anti-coagulation. Instead, noninvasive hemodynamic monitoring should be considered when managing patients with LVADs (1).

Recently, our team demonstrated that the slope of the flow waveform at the ventricular filling phase, which is displayed on the monitor of the HeartWare LVAD (Medtronic, Framingham, USA), had a strong association with the actual measured PCWP (16). Given how the pump flow depends on the pressure gradient between the left ventricle and the aorta, the increases in the left ventricular filling pressure seen in decompensated HF cases would reduce the pressure gradient in the pump, resulting in an increased flow (Fig. 3). Based on this concept, we can estimate the PCWP by measuring the slope of the HeartWare LVAD flow waveform at diastole remotely, non-invasively, and continuously.

Future smart pump designs that aim to automatically adjust pump speeds based on the needs of the individual patient will hopefully be powered by machine-derived algorithms to assess the flow waveform slope and make appropriate device-speed changes. While how far we are from the development of such a smart pump is unclear, the understanding that the HeartWare LVAD flow waveform provides valuable hemodynamic information is an important step forward. The concomitant use of a CardioMEMSTM and/or ReDS may also be a future possibility to better optimize the hemodynamic profile of patients with LVADs.

The authors state that they have no Conflict of Interest (COI).

\section{References}

1. Imamura T, Chung B, Nguyen A, Sayer G, Uriel N. Clinical implications of hemodynamic assessment during left ventricular assist device therapy. J Cardiol 71: 352-358, 2018.

2. Binanay C, Califf RM, Hasselblad V, et al. Evaluation study of congestive heart failure and pulmonary artery catheterization effectiveness: the ESCAPE trial. JAMA 294: 1625-1633, 2005.

3. Cooper LB, Mentz RJ, Stevens SR, et al. Hemodynamic predictors of heart failure morbidity and mortality: fluid or flow? J Card Fail 22: 182-189, 2016.

4. Abraham WT, Adamson PB, Bourge RC, et al. Wireless pulmonary artery haemodynamic monitoring in chronic heart failure: a randomised controlled trial. Lancet 377: 658-666, 2011.

5. Loh JP, Barbash IM, Waksman R. Overview of the 2011 Food and Drug Administration Circulatory System Devices Panel of the Medical Devices Advisory Committee Meeting on the CardioMEMS Champion Heart Failure Monitoring System. J Am Coll Cardiol 61: 1571-1576, 2013.

6. Ponikowski P, Voors AA, Anker SD, et al. 2016 ESC Guidelines for the diagnosis and treatment of acute and chronic heart failure: The Task Force for the diagnosis and treatment of acute and chronic heart failure of the European Society of Cardiology (ESC) Developed with the special contribution of the Heart Failure Association (HFA) of the ESC. Eur Heart J 37: 2129-2200, 2016.

7. Imamura T, Chung B, Nguyen A, et al. Decoupling between diastolic pulmonary artery pressure and pulmonary capillary wedge pressure as a prognostic factor after continuous flow ventricular assist device implantation. Circ Heart Fail 10: e003882, 2017.

8. Abraham WT, Stevenson LW, Bourge RC, et al. Sustained efficacy of pulmonary artery pressure to guide adjustment of chronic heart failure therapy: complete follow-up results from the CHAMPION randomised trial. Lancet 387: 453-461, 2016.

9. Costanzo MR, Stevenson LW, Adamson PB, et al. Interventions linked to decreased heart failure hospitalizations during ambulatory pulmonary artery pressure monitoring. JACC Heart Fail 4: 333-344, 2016.

10. Amir O, Azzam ZS, Gaspar T, et al. Validation of remote dielectric sensing (ReDS) technology for quantification of lung fluid status: comparison to high resolution chest computed tomography in patients with and without acute heart failure. Int J Cardiol 221: 841-846, 2016.

11. Uriel N, Sayer G, Imamura $T$, et al. Relationship between noninvasive assessment of lung fluid volume and invasively measured cardiac hemodynamics. J Am Heart Assoc 7: e009175, 2018.

12. Amir O, Ben-Gal T, Weinstein JM, et al. Evaluation of remote dielectric sensing (ReDS) technology-guided therapy for decreasing heart failure re-hospitalizations. Int J Cardiol 240: 279-284, 2017.

13. Imamura $T$, Jeevanandam V, Kim G, et al. Optimal hemodynamics during left ventricular assist device support are associated with reduced readmission rates. Circ Heart Fail 12: e005094, 2019.

14. Imamura $T$, Nguyen A, Kim G, et al. Optimal haemodynamics during left ventricular assist device support are associated with reduced haemocompatibility-related adverse events. Eur J Heart Fail 21: 655-662, 2019.

15. Uriel N, Burkhoff D, Rich JD, et al. Impact of hemodynamic ramp test-guided HVAD speed and medication adjustments on clinical outcomes. Circ Heart Fail 12: e006067, 2019. 
16. Grinstein J, Rodgers D, Kalantari S, et al. HVAD waveform analysis as a noninvasive marker of pulmonary capillary wedge pressure: a first step toward the development of a smart left ventricular assist device pump. ASAIO J 64: 10-15, 2018.
The Internal Medicine is an Open Access journal distributed under the Creative Commons Attribution-NonCommercial-NoDerivatives 4.0 International License. To view the details of this license, please visit (https://creativecommons.org/licenses/ by-nc-nd/4.0/).

(C) 2021 The Japanese Society of Internal Medicine Intern Med 60: 167-171, 2021 